\title{
Conduction mechanisms in carbon matrix containing ferromagnetic nanoparticles
}

\author{
E.P.Sajitha*, V.Prasad, S.V.Subramanyam \\ Department of Physics, Indian Institute of Science, Bangalore, India, 560012
}

\begin{abstract}
We have investigated the low temperature resistance and magnetoresistance behaviour of the carbon matrix containing ferromagnetic nanoparticles. A range of compositions with different levels of disorder and varying iron content were prepared from the pyrolysis of organic precursor maleic anhydride and ferrocene. The temperature dependence of resistivity shows a negative temperature coefficient in the entire measurement range of 1.3 to $300 \mathrm{~K}$. An increase in conductivity with the external magnetic field and a characteristic upturn at low temperature, is seen in all the compositions. The zero-field conductivity and the magnetoresistance can be explained with in the framework of two-dimensional electron-electron interaction model and the Kondo-like behaviour.
\end{abstract}

Key words:

Localization conductivity, Magnetoresistance, Kondo effect

PACS: 72.21.-i, 72.20 My, 72.15.Qm

\section{Introduction}

Quantum transport phenomena have been intensively investigated in mesoscopic metallic systems. For non-magnetic systems the resistance acquires a logarithmic temperature dependence, which is clearly proven to result from weak localization (WL) and/or enhanced electron-electron interaction (EEI) effects in two dimensional systems rather than the Kondo effect. Weak localization in disordered medium results from constructive interference of partially backscattered electron waves traveling back along time reversed paths and is essentially a low temperature phenomenon $[1,2]$. At higher temperature

* corresponding author Tel.: +91-80-2293-2313; fax: +91-80-2360-2602.

Email address: sajitha@physics.iisc.ernet.in (E.P.Sajitha). 
inelastic scattering processes due to electron-phonon or electron-electron interactions destroys the phase coherence between the carrier waves. The EEI effects arise from modified screening of the Coulomb interaction of interacting electrons due to diffuse scattering [1]. Distinction between the two important quantum mechanical phenomenon is done by the application of an external magnetic field. In the systems governed by the WL effect, small magnetic fields $(\sim B=0.01 \mathrm{~T})$ are sufficient to partially destroy the phase coherence between electron waves, leading to a low-field negative magnetoresistance (MR) in the absence of spin-orbit interactions. On the other hand, small external magnetic fields hardly has any effect on the EEI and the MR due to EEI effect occurs at larger magnetic fields.

In the case of metallic systems containing ferromagnetic material, the internal magnetic field inside the sample may or may not destroy the phase coherence of the backscattered electron waves [3]. Most of the work on ferromagnetic thin film shows EEI effect as the predominant quantum transport phenomenon [4] where as for the pyrolytic graphite and other carbon based materials, two-dimensional WL effect is seen at low temperature [5-7]. When magnetic impurity are present in a metal, the impurity spin interacts with the conduction electron gas producing an anomaly in the resistance known by classic "Kondo" effect [8]. As the temperature is lowered the conduction electron screens out the impurity moment with increasing efficiency leading to a logarithmic resistance behaviour and a negative magnetoresistance, caused by the freezing out of spin-flip scattering due to the alignment of impurity spins by the magnetic field [9]. When the density of magnetic impurities is high, the spin-ordering tendencies due to interaction between impurities can no longer be ignored. The two competing effects that lead to two different tendencies of the system to interact with conduction electrons-the Kondo effect and the Ruderman-Kittel-Kasuya-Yoshida (RKKY) interaction $[10,11]$ have a crucial influence on the impurity magnetism. The conduction electron-mediated, RKKY indirect-exchange interaction favors magnetic ordering of impurities, whereas the Kondo effect tends to quench individual impurity spins. The logarithmic increase in resistivity as the temperature decreases, is characteristic of a Kondo lattice system.

In this paper we try to understand the interplay of localization and Kondo effect in the ferromagnetic disordered system. The carbon matrix containing ferromagnetic nanoparticles were prepared from pyrolysis of organic precursors [12]. The characterization of the powder composition by X-ray diffraction, Mössbauer spectroscopy and magnetization measurement [12-14] revealed the phase composition of ferromagnetic iron carbide nanoparticles. The main aim in the present investigation is to study the conduction mechanism of the compositions with varying concentration of iron and different level of disorder in the semiconducting carbon matrix. 


\section{Experiment}

The measurements described below were carried out on the compositions (C:Fe) prepared by pyrolyzing maleic anhydride together with ferrocene in different weight percentages. The preparations were carried out in a home built temperature controlled furnace at $980{ }^{\circ} \mathrm{C}$ in a quartz tube. Full details of the preparation procedure are given elsewhere [12]. The powdery samples scratched out from the tube surfaces were pelletized under the pressure of $500 \mathrm{psi}(\sim 3447 \mathrm{kPa})$ for the time duration of $20 \mathrm{~min}$. The pellets of $8 \mathrm{~mm}$ diameter and $1.2 \mathrm{~mm}$ thickness were used for the resistivity and magnetoresistance measurements. The measurements presented here are on the compositions prepared from 5\%,10\%, 20\% and 50\% ferrocene, containing 4.6\%, $8.6 \%, 9.1 \%$ and $11.2 \%$ Fe respectively. A conventional dc four-probe method was used with silver paste contacts for measurements in a superconducting cryostat, in the temperature range of 1.3 and $300 \mathrm{~K}$ and a magnetic field up to $7 \mathrm{~T}$ perpendicular to the sample surface. The diffraction peak revealed the graphitization of carbon matrix and the interlayer spacing calculated from the mosaic spread, i.e., full width at half maximum of the (002) line, were found to vary from $3.424 \stackrel{A}{A}$ for lowest Fe content sample to $3.367 \stackrel{\AA}{A}$ for the highest Fe content sample, indicating their turbostratic nature $[13,14]$.

\section{Results and Discussion}

\subsection{Localization and Kondo Effect}

The scaling theory of localization is universal in describing the conductivity mechanism in various disordered metallic systems. In semimetallic systems the temperature dependent correction terms arising from localization and interaction are additive, to the drude conductivity for lower orders. The zero field two dimensional (2D) conductivity shows a logarithmic dependence on temperature i.e.,

$$
\sigma_{2 D}(T)=\sigma\left(T_{o}\right)+\frac{e^{2}}{2 \hbar \pi^{2}}\left[\alpha p+\left(1-\frac{3}{4} \tilde{F}_{\sigma}\right)\right] \ln \left[\frac{T}{T_{o}}\right]
$$

For an orbitally non-degenerate free electron gas, $\alpha$ is close to 1 . The Thouless length is given by $L_{T h}=\left(D \tau_{i n}\right)^{1 / 2}, D$ is the diffusion constant and $\tau_{\text {in }} \propto$ $T^{-p}, p>1$, is the inelastic scattering time. The value of $p=3 / 2,2$, and 3 depending on whether electron-electron scattering is in dirty limit, clean limit or electron-phonon dominates the inelastic scattering rate respectively [1]. The

Coulomb interaction term is $\left(1-\frac{3}{4} \tilde{F}_{\sigma}\right)$, where the size of the Hartree term 
$\tilde{F}_{\sigma}$ gives the electron screening length. For many of the disorder system, at high temperature a cross over to three dimensional weak localization (3DWL), $\sigma(T) \propto T^{p / 2}$ is observed.

Future, we incorporate the Kondo-formula to understand the effect of magnetic species. Kondo [8] suggested the contribution of extra $s-d$ exchange interaction to the temperature dependence of zero-field resistivity by the following equation for $T>T_{K}$ :

$$
\begin{aligned}
\Delta \rho= & \frac{3 c}{\pi e^{2} v_{F}^{2}} \frac{\Omega}{N}\left\{\sin ^{2} \eta-2 \pi^{2} J^{2} \rho_{1}^{2} S(S+1) \cos ^{4} \eta \sin ^{2} \eta\right. \\
& \left.+\pi^{2} J^{2} \rho_{1}^{2} S(S+1) \frac{\cos ^{4} \eta \cos 2 \eta}{\left[1-2 \tilde{J} \rho_{1} \ln \left(\frac{T}{D_{\varepsilon}}\right)\right]^{2}}\right\}
\end{aligned}
$$

where $\eta$ is the phase shift due to the potential scattering given as $\tan \eta=-\pi \mathrm{V} \rho_{1}$ and $\rho_{1}$ is the carrier concentration per one spin degree of freedom near the Fermi energy and $V$ the magnitude of the potential energy. $\Omega$ is the volume of the host metal, $S$ the effective spin angular moment of unpaired spin in the existing magnetic impurities, $D_{\varepsilon}$ the cutoff energy which corresponds to

the Fermi energy and $\tilde{J}$ the effective exchange interaction integral given as $\tilde{J}=J \cos ^{2} \eta$. The positive (negative) $J$ value corresponds to the ferromagnetic (antiferromagnetic) $s-d$ exchange coupling between the conduction electrons and the localized moments.

\subsection{Magnetoconductance}

The mechanism underlying the conductance regimes are further evidenced by the behaviour of magnetoconductance. In the framework of the 2DWL theory, the negative magnetoconductance is given by $[2,3]$,

$$
\begin{aligned}
M C=\frac{e^{2}}{2 \hbar \pi^{2} G(0, T)}[ & -\Psi\left[\frac{1}{2}+\frac{B_{1}}{B}\right]+\frac{3}{2} \Psi\left[\frac{1}{2}+\frac{B_{2}}{B}\right]-\frac{1}{2} \Psi\left[\frac{1}{2}+\frac{B_{3}(T)}{B}\right] \\
& \left.-\ln \frac{B_{2}^{3 / 2}}{B_{1} B_{3}^{1 / 2}}\right]
\end{aligned}
$$

where $\Psi$ is the digamma function and $B_{1}, B_{2}(\mathrm{~T})$ and $B_{3}(\mathrm{~T})$ are defined by $B_{1}=B_{0}+B_{\text {s.o }}+B_{s}$ $B_{2}(T)=B_{i}(T)+\frac{4}{3} B_{\text {s.o }}+\frac{2}{3} B_{s}$ $B_{3}(T)=B_{i}(T)+2 B_{s}$ where the quantities $B_{k}=\frac{\hbar}{4 e D \tau_{k}}$ represent the characteristic magnetic fields 
associated with the scattering mechanism, $k$ : elastic scattering (0), inelastic scattering $(i)$, magnetic impurity scattering $(s)$, or spin-orbit coupling (s.o); is the relaxation time corresponding to the scattering type ' $k^{\prime}$ and $D$ is the diffusion constant defined by $D=\frac{1}{2} \vartheta_{F}^{2} \tau_{k}$, where $\vartheta_{F}$ is the Fermi velocity.

The component of magnetoconductance due to 2D interaction effect, or splitting of electron energies into spin up and spin down bands, when the condition $h=g \mu_{B} B / k_{B} T \gg 1$ is satisfied (where $g$ is the $g$ factor, $\mu_{B}$ the Bohr magneton, and $k_{B}$ the Boltzmann constant) comprises of two terms [15].

$$
\delta \sigma_{I}(B, T)=\delta \sigma_{I}^{\prime}(T)+\delta \sigma_{I}^{\prime \prime}(B, T)
$$

In the presence of magnetic field, $\delta \sigma_{I}^{\prime \prime}(B) \propto \ln (B)$ for $h \gg 1$ and $\delta \sigma_{I}^{\prime \prime}(B) \propto B^{2}$ for $h \ll 1$. The MR arising from spin-splitting of the conduction-electron energies are mainly positive.

In Kondo-effect, the negative magnetoresistance could originate due to the depression of the spin-flip scattering [9].

For $h \ll 1$

$$
\begin{aligned}
\Delta \rho \cong-\frac{3 \pi}{2 \epsilon_{F}} & \frac{m}{e^{2} \hbar} \frac{c v_{0}}{9}\left(\frac{g \mu_{B} B}{k_{B} T}\right)^{2} J^{2} S(S+1)\{1+4 S(S+1) \\
& \left.+\frac{3 z J}{\epsilon_{F}}\left[(1+4 S(S+1)) \ln \frac{k_{B} T}{2 \epsilon_{F}}+6.27 S(S+1)+1.11\right]\right\}
\end{aligned}
$$

i.e. $\Delta \rho \propto B^{2}$

and for $h \gg 1$

$$
\begin{aligned}
\Delta \rho \cong-\frac{3 \pi}{2 \epsilon_{F}} \frac{m}{e^{2} \hbar} c v_{0}\left\{V^{2}+\right. & J^{2}\left[S(S+1)-4\left\langle S_{z}\right\rangle^{2}-\left\langle S_{z}\right\rangle\right] \\
\times & {\left.\left[1+\frac{3 z J}{\epsilon_{F}}\left(2+\ln \frac{g \mu_{B} B}{k_{B} T}\right)\right]\right\} }
\end{aligned}
$$

i.e. $\quad \Delta \rho \propto \ln B$

where $v_{0}$ is the atomic volume of the host metal, $c$ the impurity concentration and $z$ the number of conduction electrons per atom. The $\ln B$ term reflects Kondo-type anomalous behaviour in the applied field when all the spins are aligned by the magnetic field, so that $\left\langle S_{z}\right\rangle$ is a constant. 


\subsection{Fitting of the conductivity data}

The overall temperature dependence of resistivity for the prepared compositions are shown in Fig.1. At first we considered the general localization formula

$$
\sigma(T)=\sigma\left(T_{o}\right)+\mathrm{A} \ln \left(\frac{T}{T_{0}}\right)+\mathrm{B} T^{1.5}
$$

with $A, B, \sigma\left(T_{o}\right)$ and $T_{o}$ as the fitting parameters, to fit the zero field conductivity data. The model fits very well as shown in Fig. 2. The last term in the above equation corresponds to the electron-phonon dominant inelastic scattering in 3DWL. The fitted parameters are shown in Table 1. For the composition with $4.6 \% \mathrm{Fe}$ and $9.2 \% \mathrm{Fe}$ no dimensionality crossover is observed. The strong temperature dependence of conductivity mainly at low temperature, indicates that 2DWL may not be the prominent mechanism of conduction in these compositions. This is further evidenced when a fit to the magnetoconductance data, where carried out using Eq.3. The fitting parameters $B_{2}(T)$ and $B_{3}(T)$ showed very weak temperature dependence.

Second, we tried to fit the zero-field resistivity with the Kondo effect formula [Eq. 2], incorporating the effect of well-dispersed iron and iron carbide nanoparticles on the conductivity behaviour, as shown in Fig. 3. The Kondo formula fits well for all the four compositions. The resultant fitting parameters are given in the Table 2 . The positive $J$ value refers to the ferromagnetic $s-d$ exchange coupling between conduction electrons and the localized moments. Even though the temperature dependence of magnetic properties of the nanoparticle compositions shows antiferromagnetic interaction $[13,14]$ between the localized carbon spin, signalling RKKY type interaction mediated by the ferromagnetic clusters, conduction mechanism is mainly dominated by the Kondo-type interaction.

The magnetic field dependence of resistivity at different temperature for the composition with $4.6 \%$ and $8.6 \%$ Fe are shown in Fig. 4. At high temperature very small negative MR is seen. The effect of EEI diminishes the MR values at high temperature and results in a positive MR at low temperature. The MR plot for the compositions with $9.1 \%$ Fe and $11.2 \%$ Fe are shown in Fig. 5 and Fig. 6. No change in resistance with the applied magnetic field is seen for temperatures above $75 \mathrm{~K}$. Below $75 \mathrm{~K}$, negative MR is seen with a characteristic upturn at low temperature. The positive upturn occurs at an applied field of about $5 \mathrm{~T}$ at $4.2 \mathrm{~K}$ and $6 \mathrm{~K}$ for the $9.1 \%$ and $11.2 \% \mathrm{Fe}$ composition respectively. The MR plot fits well to the $\ln B$ dependence at high magnetic field and $B^{2}$ dependence at low field in all the temperature range of measurement expect at the characteristic upturn. 


\section{Summary}

The zero field resistivity fits well to the Kondo effect formula. A shift to 2D EEI is seen at low temperatures. The negative magnetoresistance, with a characteristic positive upturn at high magnetic field and low temperature, has been understood within the frame work of these two models. The extend of disorder in the matrix and the magnetic nanoparticle interaction with the conduction electrons plays an important role in governing the conduction mechanism of our compositions.

\section{Acknowledgments}

We express our deep gratitude to all the present and past colleagues for their support during the experimental work. Financial support by DST and CSIR is gratefully acknowledged.

\section{References}

[1] P.A. Lee, and T. V. Ramakrishnan, Rev. Mod. Phys.,57, (1985) 287

[2] G. Bergmann, Phys. Rep.,107, (1984) 1,

[3] G. Bergmann, Phys. Rev. Lett., 49,(1982) 162,

[4] M. Brands, A. Carl, O. Posth, and G. Dumpich, Phys. Rev. B, 72, (2005) 085457

[5] V. Bayot, L. Piraux, J.P. Michenaud, J.P. Issi, M. Lelaurain and A.Moore, Phys. Rev. B, 41, (1990) 11770

[6] V. Bayot, L. Piraux, J.-P. Michenaud, and J.-P. Issi, Phys. Rev. B, 40, (1989) 3514

[7] A.A. Bright, Phys. Rev. B, 20, (1979) 5142

[8] J. Kondo, Phys. Rev., 169, (1968) 437

[9] M.-T. Beal-Monod and R.A. Weiner, Phys. Rev.,170, (1968) 552

[10] B.A. Jones, C.M. Varma and J.W. Wilkins, Phys. Rev. Lett., 61, (1988) 125

[11] C. Jayaprakash, H.R. Krishnamurthy and J.W. Wilkins, Phys. Rev. Lett. 47, (1981) 737

[12] E.P. Sajitha, V. Prasad, S.V. Subramanyam, S. Eto, Kazuyuki Takai and T.Enoki, Carbon, 42, (2004) 2815

[13] E.P.Sajitha, S. Shekhar, V.Prasad, S.V.Subramanyam, A. K. Mishra, S. Sarkar and C. Bansal, Submitted to J. Phys.: Condens. Matter. 
[14] E.P.Sajitha, V.Prasad, S.V.Subramanyam, A. K. Mishra, S. Sarkar and C. Bansal, in manuscript

[15] P.A. Lee, T.V. Ramakrishnan, Phys. Rev. B, 26, (1982) 4009 
Table 1

The parameters obtained by fitting the localization formula to the temperature dependence of conductivity using Eq. 7 .

\begin{tabular}{ccccc}
\hline$F e \%$ & $\sigma\left(T_{o}\right)$ & $A$ & $T_{0}$ & $B$ \\
\hline $4.6^{a}$ & $235.7(8)$ & $41.18(8)$ & $86.53(2)$ & - \\
8.6 & $129.3(9)$ & $64.69(2)$ & $1.97(6)$ & $0.059(5)$ \\
9.1 & $111.8(3)$ & $15.19(1)$ & $259.53(8)$ & - \\
11.2 & $126.2(9)$ & $29.11(7)$ & $14.56(8)$ & $0.025(5)$ \\
\hline
\end{tabular}

a. In the temperature range $1.3 \mathrm{~K}-100 \mathrm{~K}$

Table 2

The parameters obtained by fitting the Kondo formula to the temperature dependence of resistivity.

\begin{tabular}{ccccc}
\hline$F e \%$ & $\eta$ & $J \rho_{1}$ & $S$ & $D_{\epsilon}(m K)$ \\
\hline 4.6 & $\pi / 18.35$ & 0.602 & 0.116 & 24.5 \\
8.6 & $\pi / 6.298$ & 1.134 & 0.062 & 49.4 \\
9.1 & $\pi / 6.300$ & 1.135 & 0.062 & 16.3 \\
11.2 & $\pi / 6.309$ & 1.136 & 0.062 & 0.76 \\
\hline
\end{tabular}

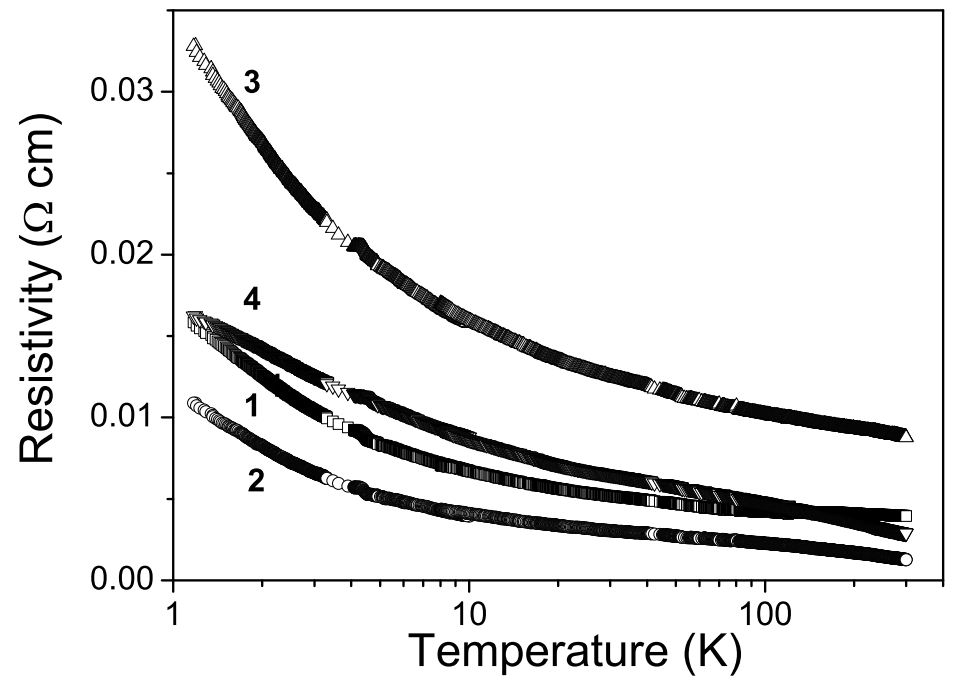

Fig. 1. The temperature dependence of zero-field resistivity for the (1) $4.6 \%$, (2) $8.6 \%,(3) 9.1 \%$ and (4) $11.2 \%$ Fe composition. 


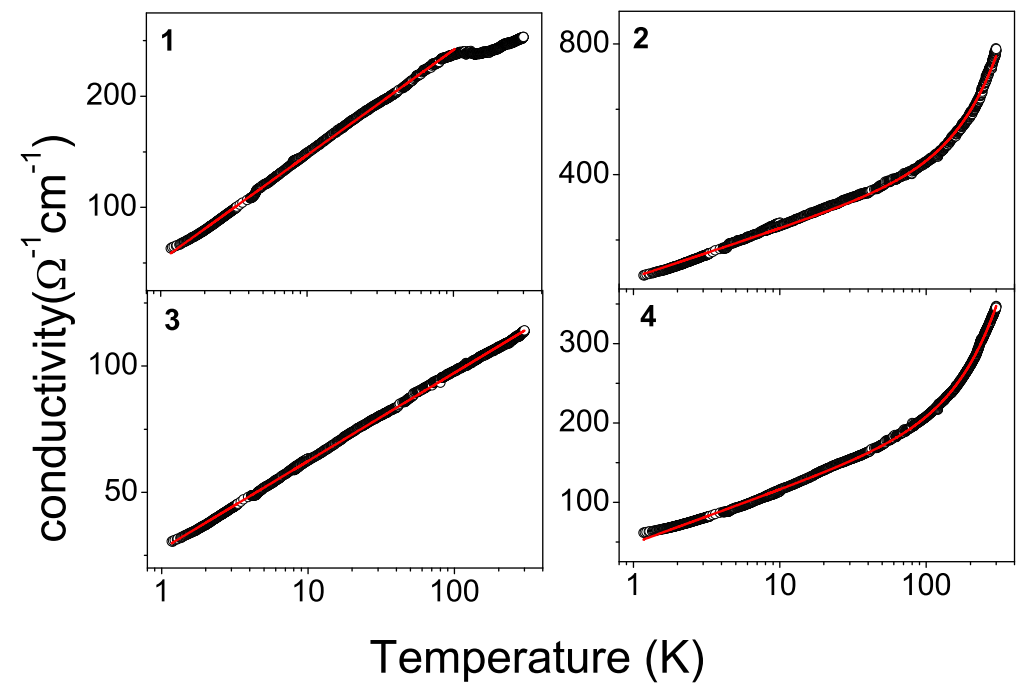

Fig. 2. The temperature dependence of zero-field conductivity fitted to the general formula [Eq.7] for the (1) $4.6 \%$, , (2) $8.6 \%$, (3) $9.1 \%$ and (4) $11.2 \%$ Fe composition.

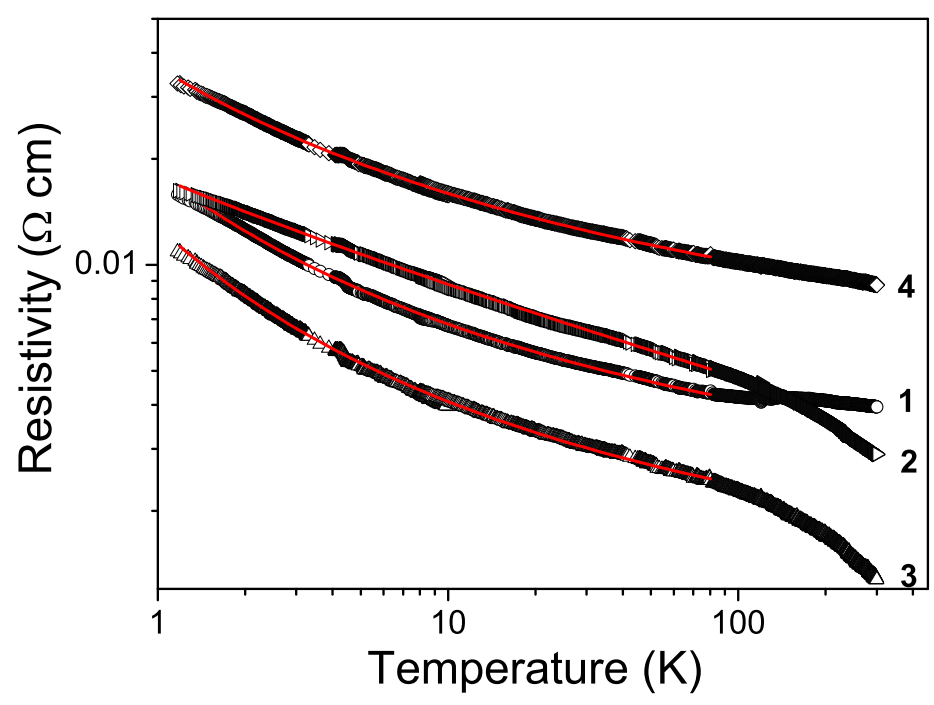

Fig. 3. The temperature dependence of zero-field resistivity fitted to the kondo equation for the (1) $4.6 \%$, , (2) $8.6 \%$, (3) $9.1 \%$ and (4) $11.2 \%$ Fe composition. 


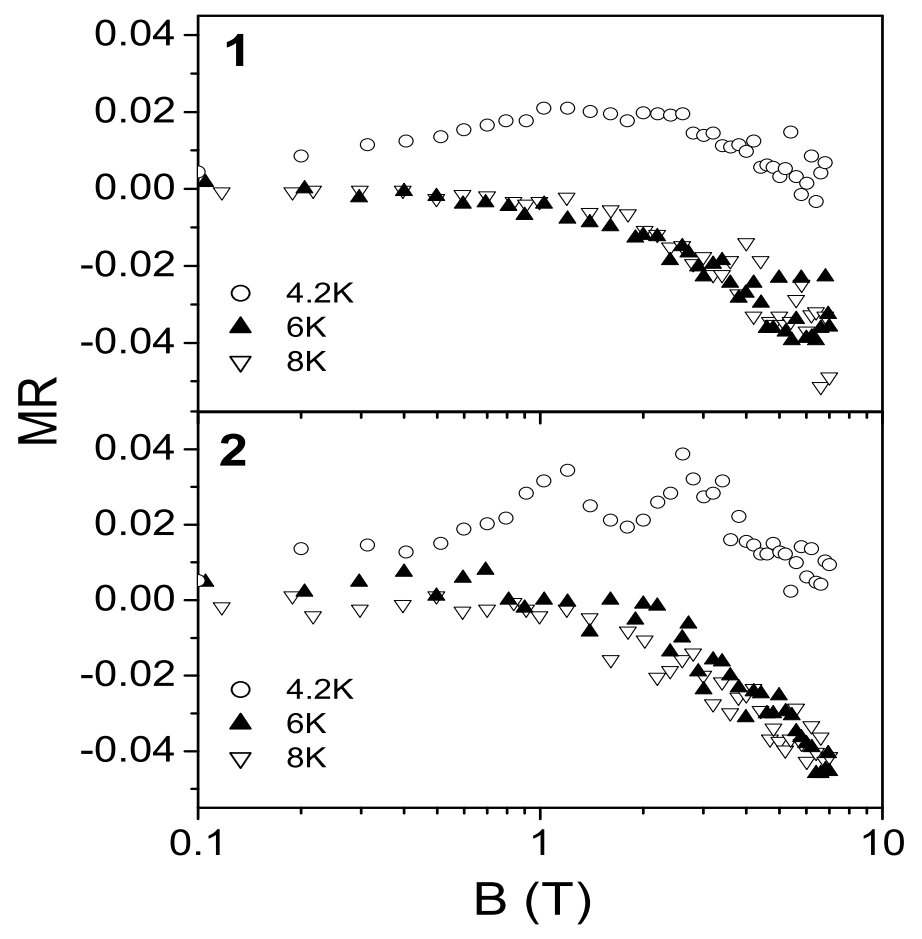

Fig. 4. The low temperature magnetoresistance plot for the (1)4.6\% and (2) $8.6 \%$ Fe composition at $\mathrm{T}=4.2,6$ and $8 \mathrm{~K}$. 


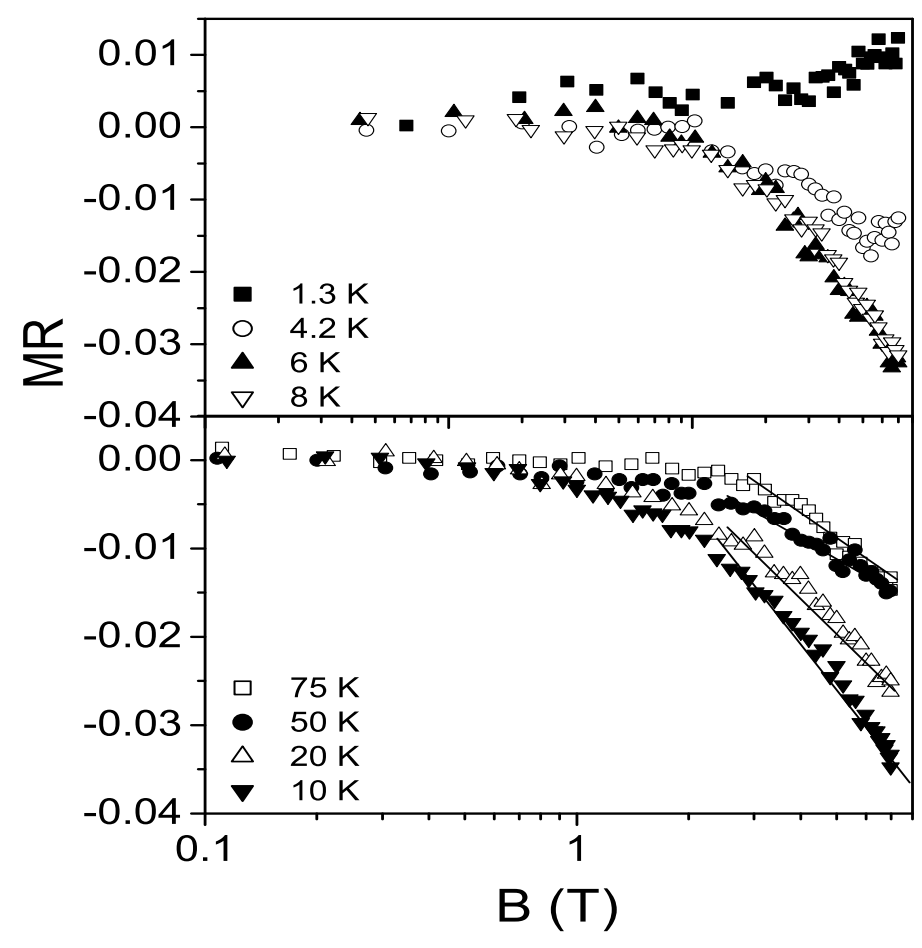

Fig. 5. The magnetoresistance plot for the $9.1 \%$ Fe composition at $\mathrm{T}=1.3,4.2,6$, $8,10,20,50$ and $75 \mathrm{~K}$. The straight line indicates the $\ln B$ dependence at high field. 


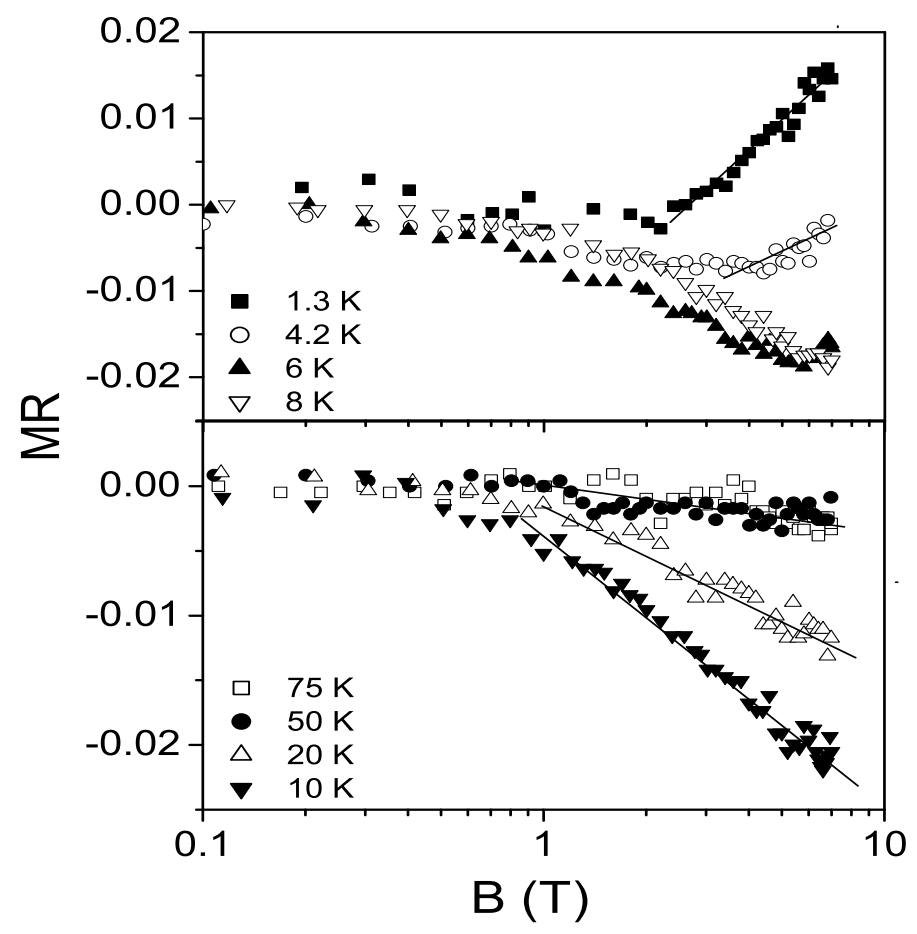

Fig. 6. The magnetoresistance plot for the $11.2 \%$ Fe composition at $\mathrm{T}=1.3,4.2,6$, $8,10,20,50$ and $75 \mathrm{~K}$. The straight line indicates the $\ln B$ dependence at high field. 